\title{
Interaction-induced effects in the nonlinear coherent response of quantum-well excitons
}

Wagner, Hans Peter; Schätz, A.; Langbein, Wolfgang Werner; Hvam, Jørn Märcher; Smirl, Arthur L.

Published in:

Physical Review B

Link to article, DOI:

10.1103/PhysRevB.60.4454

Publication date:

1999

Document Version

Publisher's PDF, also known as Version of record

Link back to DTU Orbit

Citation (APA):

Wagner, H. P., Schätz, A., Langbein, W. W., Hvam, J. M., \& Smirl, A. L. (1999). Interaction-induced effects in the nonlinear coherent response of quantum-well excitons. Physical Review B, 60(7), 4454-4457.

https://doi.org/10.1103/PhysRevB.60.4454

\section{General rights}

Copyright and moral rights for the publications made accessible in the public portal are retained by the authors and/or other copyright owners and it is a condition of accessing publications that users recognise and abide by the legal requirements associated with these rights.

- Users may download and print one copy of any publication from the public portal for the purpose of private study or research.

- You may not further distribute the material or use it for any profit-making activity or commercial gain

- You may freely distribute the URL identifying the publication in the public portal 


\title{
Interaction-induced effects in the nonlinear coherent response of quantum-well excitons
}

\author{
H. P. Wagner \\ Institut für Physik, TU Chemnitz, 09107 Chemnitz, Germany
}

A. Schätz

Institut Physik II, Universität Regensburg, D-93040 Regensburg, Germany

W. Langbein and J. M. Hvam

Mikroelektronik Centret, The Technical University of Denmark, 2800 Lyngby, Denmark

Arthur L. Smirl

\author{
Laboratory for Photonics and Quantum Electronics, 100 IATL, University of Iowa, Iowa City, Iowa 52242
}

(Received 5 November 1998; revised manuscript received 3 March 1999)

\begin{abstract}
Interaction-induced processes are studied using the third-order nonlinear polarization created in polarizationdependent four-wave-mixing experiments (FWM) on a ZnSe single quantum well. We discuss their influence by a comparison of the experimental FWM with calculations based on extended optical Bloch equations including local-field effects, excitation-induced dephasing, and biexciton formation. The investigations show that, for copolarized input fields, excitation-induced dephasing is the dominant FWM mechanism, followed by the conventional density-grating FWM process, biexcitonic contributions, and local-field effects. For crosslinear polarized input fields the excitation-induced dephasing mechanism is canceled so that the conventional density-grating FWM process and biexcitonic contributions are dominating. [S0163-1829(99)05428-4]
\end{abstract}

Ultrafast degenerate four-wave mixing (FWM) techniques have been applied successfully to study the fundamental quantum coherent processes in bulk semiconductors and quantum well (QW) structures. A general theoretical approach to analyze these coherent optical phenomena is based on the semiconductor Bloch equations (SBE), which consider the fermionic nature and the Coulomb interaction of the carriers in a semiconductor. However, under certain excitation conditions the important mechanisms can be obtained also if the complexity of the SBE is simplified by phenomenological models, essentially representing extended optical Bloch equations (OBE). These models consider, e.g., localfield effects (LFE), ${ }^{1}$ excitation-induced dephasing ${ }^{2-4}$ (EID) and biexciton formation (BIF). 5,6

In this paper, we investigate the relative importance of these many-body effects by polarization-dependent degenerate FWM experiments on a $\mathrm{ZnSe}$ single QW (SQW). Since $\mathrm{ZnSe}$ possesses an exciton oscillator strength which is about 10 times higher than that of GaAs, this SQW structure is well suited for the study of the mentioned coherent phenomena. The higher biexciton binding energy in II-VI semiconductors compared to III-V semiconductors also enables a spectral isolation of the BIF process. ${ }^{7-10}$ Using a SQW, interference between different QW's (Ref. 11) and propagation effects vertical to the sample surface, as observed in optically thick layers ${ }^{12}$ are excluded.

We investigate the dominantly homogeneously broadened lowest excitonic transition in a high-quality $\mathrm{ZnSe} \mathrm{SQW}$ pseudomorphically grown on (001) GaAs by molecularbeam epitaxy. The SQW has a thickness of $10 \mathrm{~nm}$ and is sandwiched between two $25-\mathrm{nm}$ thick $\mathrm{Zn}_{0.9} \mathrm{Mg}_{0.1}$ Se barriers, defining a type I structure. A frequency-doubled, modelocked Ti-sapphire laser was used as excitation source, producing 100 fs pulses (full width at half maximum irradiance) of $22 \mathrm{meV}$ spectral width at a repetition rate of $76 \mathrm{MHz}$. The FWM is performed in reflection geometry using a two-pulse degenerate configuration. The FWM signal is detected in the direction $2 \mathbf{k}_{2}-\mathbf{k}_{1}$ time-integrated and spectrally resolved by a combination of a spectrometer and an optical multichannel analyzer, as function of the time delay $\tau$ between the two incident pulses in the directions $\mathbf{k}_{1}$ and $\mathbf{k}_{2}$. The sample was kept in a helium cryostat at a temperature of $50 \mathrm{~K}$.

In the investigated SQW, the biaxial strain and the twodimensional (2D) confinement gives rise to a series of $1 \mathrm{~s}$ excitons below the continuum, which are identified as the heavy-hole $(11 h, 13 h)$ and light-hole $(11 l)$ exciton states, as discussed in Ref. 13. A coherent excitation of these excitons leads to pronounced quantum beats (QB) described in Ref. 14. In the following, we have tuned the center laser wavelength energetically below the lowest excitonic transition $(11 h)$ in order to minimize the excitation of higher exciton states and excitonic continua. The FWM spectra at a delay time of $\tau=-0.5$ ps for collinear $(\uparrow \uparrow)$, cross-linear $(\uparrow \rightarrow)$, and cocircular $\left(\sigma^{+}, \sigma^{+}\right)$polarized input fields are shown in Fig. 1 . The exciton density $n_{X}$ created by the excitation pulses of $2 \mathrm{MW} / \mathrm{cm}^{2}$ intensity is estimated ${ }^{14,15}$ to $n_{X} \approx 5 \times 10^{9} \mathrm{~cm}^{-2}$. Besides the $11 h$ FWM signal $(X)$, a low-energy peak $(X X)$ appears, that is attributed to the exciton-biexciton transition, according to its polarization dependence, showing a relative intensity increase in $(\uparrow \rightarrow)$ configuration and a strong suppression in $\left(\sigma^{+}, \sigma^{+}\right)$configuration. The deduced biexciton binding energy is $E_{b X X}=4.7 \mathrm{meV} \pm 0.2 \mathrm{meV}$.

The FWM traces at the $X X$ and $X$ center transition energy for different input polarizations are given in Figs. 2(a) and 2(b), respectively. The intensities of the traces are normalized to the $X$ FWM trace maximum in $(\uparrow \uparrow)$ configuration. In all FWM traces, weak QB are observed, originating from the remaining excitation of the $13 h$ and $11 l$ excitons. Striking 


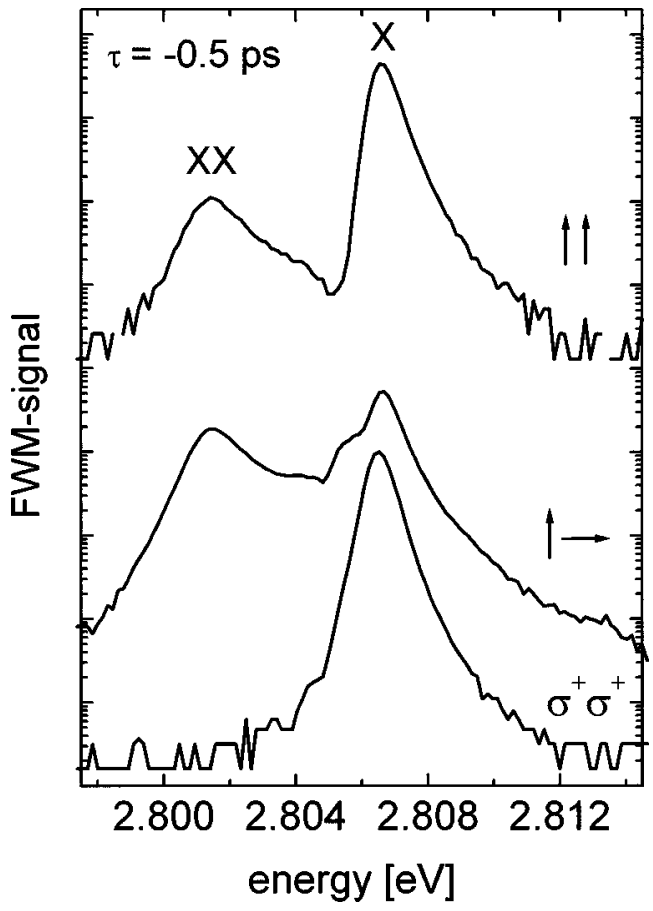

FIG. 1. FWM spectra at $\tau=-0.5$ ps for collinear $(\uparrow \uparrow)$, crosslinear $(\uparrow \rightarrow)$, and cocircular $\left(\sigma^{+}, \sigma^{+}\right)$polarized input fields obtained from the $\mathrm{ZnSe} \mathrm{SQW}$. The excitation was centered at $2.806 \mathrm{eV}$, the excitation intensity per pulse was $2 \mathrm{MW} / \mathrm{cm}^{2}$.

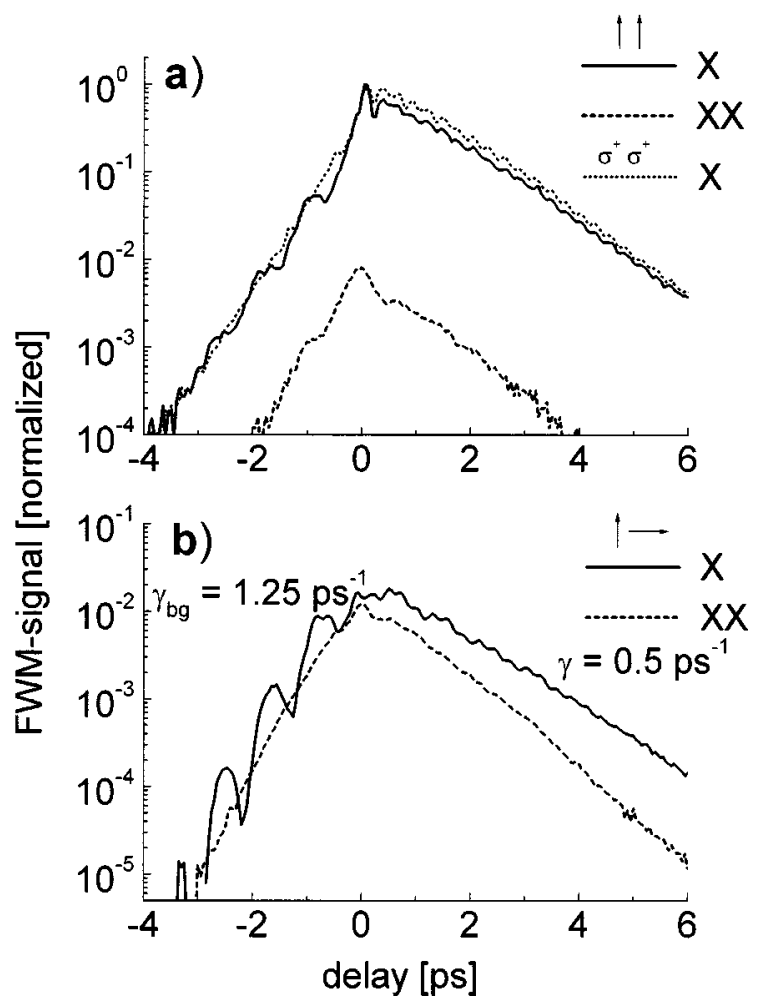

FIG. 2. (a) FWM traces at the energetic position of the $11 \mathrm{~h}$ exciton transition $(X)$ for collinearly $(\uparrow \uparrow)$ and cocircularly $\left(\sigma^{+}, \sigma^{+}\right)$-polarized fields and at the exciton-to-biexciton transition $(X X)$ for collinearly $(\uparrow \uparrow)$ polarized fields. (b) FWM traces at the energetic position of the $11 \mathrm{~h}$ exciton $(X)$ and the exciton-tobiexciton transition $(X X)$ for cross-linearly $(\uparrow \rightarrow)$ polarized fields.

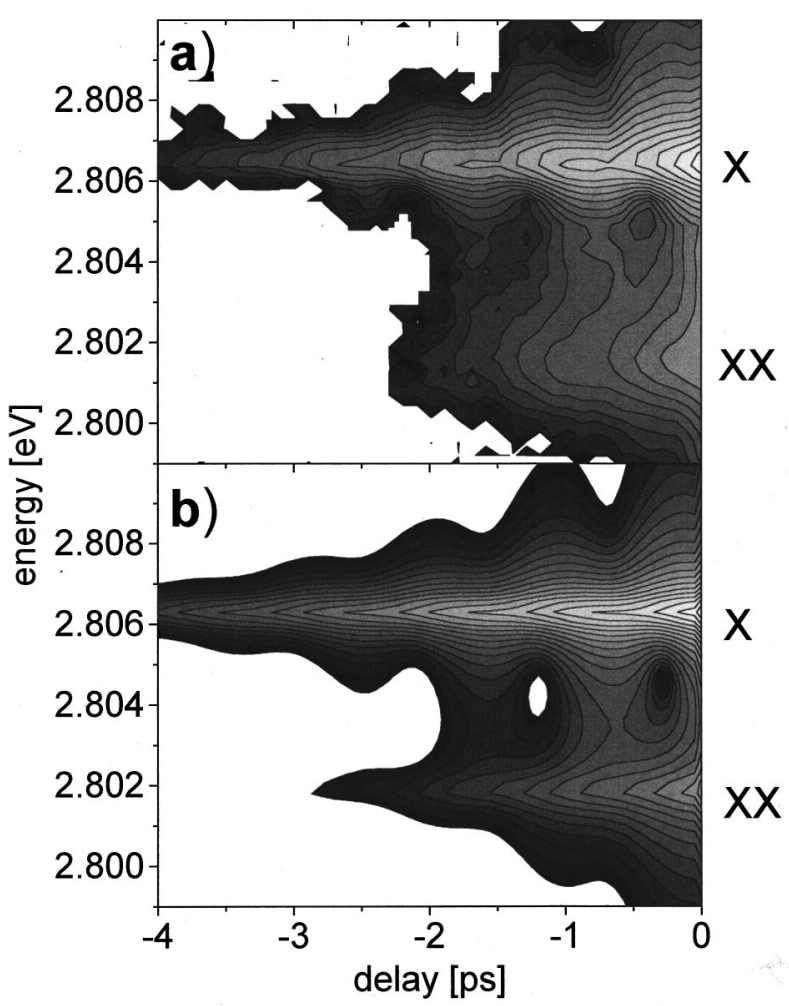

FIG. 3. (a) FWM intensity vs energy and delay time $\tau$ for ( $\uparrow$ ) polarized fields in a contour plot on a logarithmic scale over four decades. (b) Calculated spectrally resolved FWM intensity vs delay time in a contour plot on a logarithmic scale for $(\uparrow \uparrow)$ polarized fields.

features in the $X$ trace in $(\uparrow \uparrow)$ configuration at negative delay time are oscillations with a period of $T \approx 0.85 \mathrm{ps,} \mathrm{which} \mathrm{are}$ fanning out with increasing delay. The spectrally resolved FWM response given in Fig. 3(a) reveals the phase shift of these oscillations versus energy and an increase of the modulation depth with increasing energy separation from the $X$ center transition energy. These oscillations vanish in $\left(\sigma^{+}, \sigma^{+}\right)$configuration, implying that BIF is necessary for it's formation [Fig. 2(a)]. In $(\uparrow \rightarrow)$ configuration [Fig. 2(b)], where EID is inactive, the FWM signal is reduced by a factor of 40. The oscillations at negative delay time are phase shifted compared to the $(\uparrow \uparrow)$ configuration and show an increased modulation depth with increasing negative delay. The FWM signal intensity scales with the cube of the excitation intensity up to an exciton density of $n_{X}=1$ $\times 10^{10} \mathrm{~cm}^{-2}$. At higher excitation intensities the FWM signal saturates and $X-X X$ oscillations in the $X$ trace appear for positive delay times, which are clear evidence for fifth-order effects. ${ }^{16}$ For the used density the FWM signal is thus dominated by third-order processes.

To evaluate the results more quantitatively, we model the FWM response by the extended OBE. Besides a weak QB structure originating from the excitation of higher exciton states, the investigated exciton-biexciton system can be described for $\sigma^{+}$and $\sigma^{-}$transitions by a five-level system of ground, exciton, biexciton, and unbound two-exciton levels, with the ground level initially populated. The corresponding OBE are further extended to encompass LFE (Ref. 1) from first-order polarizations and EID, ${ }^{3}$ as discussed in Ref. 17. Solving this extended OBE with infinitely short excitation 
pulses, linearly polarized along $\mathbf{k}_{1} \|-\sin \theta_{12} \hat{\mathbf{e}}_{1}+\cos \theta_{12} \hat{\mathbf{e}}_{2}$, and $\mathbf{k}_{2} \| \hat{\mathbf{e}}_{1}$, the third-order polarization $P^{(3)}(t, \tau<0)$ for negative delay times $\tau=t_{2}-t_{1}$ in the $2 \mathbf{k}_{2}-\mathbf{k}_{1}$ direction reads

$$
\begin{aligned}
& P_{2 \mathbf{k}_{2}-\mathbf{k}_{1}}^{(3)}(t, \tau<0)=-i \kappa \Theta(t+\tau) \Theta(-\tau)\left\{\left[(\widetilde{\eta}+i \widetilde{\xi}) \frac{1-\exp \left(-\gamma_{e e}(t+\tau)\right)}{\gamma_{e e}} \exp [-\gamma(t+\tau)] \exp (2 \gamma \tau)+B^{2} H(t) \exp (2 \gamma \tau)\right.\right. \\
& \left.-B^{2}\{K(t)+M(t)\} \exp \left[\left(\gamma_{b g}-i \Delta\right) \tau\right]\right] \cos \theta_{12} \hat{\mathbf{e}}_{1}+\left[-i \widetilde{\xi} \frac{1-\exp \left(-\gamma_{e e}(t+\tau)\right)}{\gamma_{e e}} \exp [-\gamma(t+\tau)] \exp (2 \gamma \tau)\right. \\
& \left.\left.+B^{2} H(t) \exp (2 \gamma \tau)-B^{2}\{K(t)+M(t)\} \exp \left[\left(\gamma_{b g}-i \Delta\right) \tau\right]\right] \sin \theta_{12} \hat{\mathbf{e}}_{2}\right\} \\
& H(t)=\frac{\tilde{\xi}^{2}}{\Gamma_{1}+i \Delta}\left(\frac{\left\{1-\exp \left[-\left(\Gamma_{3}+i \Delta\right)(t+\tau)\right]\right\} \exp \left[\left(i \Delta-\gamma_{b}\right)(t+\tau)\right]}{\Gamma_{3}+i \Delta}-\frac{\{1-\exp [-2 \gamma(t+\tau)]\} \exp [-\gamma(t+\tau)]}{2 \gamma}\right) \\
& -\frac{i \widetilde{\xi}\left\{1-\exp \left[-\left(\Gamma_{4}+i \Delta\right)(t+\tau)\right]\right\} \exp \left[\left(i \Delta-\gamma_{b}\right)(t+\tau)\right]}{\Gamma_{4}+i \Delta} \\
& K(t)=\left(1+\frac{i \widetilde{\xi} \cdot\left\{1-\exp \left[\left(\Gamma_{1}+i \Delta\right) \tau\right]\right\}}{\Gamma_{1}+i \Delta}\right) \exp [-\gamma(t+\tau)]-\exp \left[-\left(\gamma_{b}-i \Delta\right)(t+\tau)\right] \\
& M(t)=\left(i \widetilde{\xi}-\frac{\widetilde{\xi}^{2}}{\Gamma_{1}+i \Delta}\right)\left(\frac{\left\{1-\exp \left[\left(i \Delta-\gamma_{b g}\right)(t+\tau)\right]\right\} \exp [-\gamma(t+\tau)]}{\gamma_{b g}-i \Delta}-\frac{\left\{1-\exp \left[-\Gamma_{2}(t+\tau)\right]\right\} \exp \left[\left(i \Delta-\gamma_{b}\right)(t+\tau)\right]}{\Gamma_{2}}\right) .
\end{aligned}
$$

$P_{2 \mathbf{k}_{2}-\mathbf{k}_{1}}^{(3)}(t, \tau>0)$ for positive delay times is given by Eqs. (8)-(10) in Ref. 17. In the equations $\Theta$ is the Heaviside function, $\hbar \Delta$ is the biexciton binding energy, $B^{2}=|\nu / \mu|^{2}$, where $\mu$ and $\nu$ are the dipole matrix elements for the groundto-exciton and exciton-to-biexciton-transition, respectively. $\kappa=2 N \mu^{4} E_{2}^{2} E_{1}^{*} / \hbar^{3} \exp (-i \Omega t)$ with $\Omega=\omega_{X}-\omega_{0}-\widetilde{\xi}$ where $\omega_{X}$ is the circular frequency corresponding to the exciton transition energy and $\omega_{0}$ is the center frequency of the laser. The dephasing rates are given by $\Gamma_{1}=2 \gamma-\gamma_{b g}, \Gamma_{2}=\gamma$ $+\gamma_{b g}-\gamma_{b}, \Gamma_{3}=3 \gamma-\gamma_{b}$, and $\Gamma_{4}=\gamma-\gamma_{b}+\gamma_{e e}$ where $\gamma$ and $\gamma_{b}$ are the dephasing rates of the exciton-to-ground and biexciton-to-exciton-transition, respectively, $\gamma_{b g}$ is the dephasing rate associated with the biexciton-to-ground-state coherence and $\gamma_{e e}=1 / T_{1}$ is the decay rate for excitons. The LFE contribution is quantified by $\widetilde{\xi}=\left(N \mu_{X}^{2} L\right) / \hbar$, with the Lorentz local field factor $L$ and the density of two level systems $N{ }^{1}$ The EID parameter $\tilde{\eta}=N \partial \gamma / \partial n$ accounts for the density-dependent dephasing rate. The dephasing rate was taken to be dependent on the total exciton density given by $n_{X} \cdot{ }^{3}$ Since there is no biexciton density up to third-order perturbation, EID effects are not present in the exciton-tobiexciton transitions. Furthermore, we have assumed that the dephasing rates and the dipole matrix elements between the unbound biexciton state and the single-exciton states are the same as for the exciton-to-ground-state transitions, i.e., equal to $\gamma$ and $\mu$, respectively. The time-integrated, spectrally resolved, third-order polarization for positive and negative delay $P_{2 \mathbf{k}_{2}-\mathbf{k}_{1}}^{(3)}(\omega, \tau)$ was derived analytically by Fouriertransforming the nonlinear polarization $P_{2 \mathbf{k}_{2}-\mathbf{k}_{2}}^{(3)}(t, \tau)$ with respect to the real time $t$.

The relative influence of the different many-body effects can now be deduced by a comparison of the experimentally observed FWM signal intensities at the exciton $\left(I_{X}\right)$ and biexciton $\left(I_{X X}\right)$ with the calculated intensities $I^{(3)}\left(\omega_{X, X X}, \tau\right) \propto\left|P_{2 \mathbf{k}_{2}-\mathbf{k}_{1}}^{(3)}\left(\omega_{X, X X}, \tau\right)\right|^{2}$ at the transition frequencies $\omega_{X, X X}$ of the ground-to-exciton $(X)$ and exciton-tobiexciton $(X X)$ transition, respectively. For negative delay times, the calculated intensity ratio in $(\uparrow \rightarrow)$ configuration is approximately given by

$$
\left.\frac{I_{X}}{I_{X X}}\right|_{\tau<0} \approx \frac{\gamma_{b}^{2}}{\gamma^{2}}\left[1+2 \widetilde{\xi} \frac{\mu^{2}}{\gamma \nu^{2}} \sin (\Delta \tau)\right]
$$

and is observed to be about 1.5 [Fig. 2(b)], giving $\gamma_{b}$ $\approx 1.2 \gamma$. The LFE parameter $\widetilde{\xi}$ is estimated to $0.2 \gamma$ from the modulation of the signal taking into account the increase of the modulation depth with increasing delay.

The calculated intensity ratio between the $X$ and $X X$ FWM-signal in $(\uparrow \rightarrow)$ configuration for positive delays is

$$
\left.\frac{I_{X}}{I_{X X}}\right|_{\tau>0} \approx \frac{\mu^{4}}{\nu^{4}} \frac{\gamma_{b}^{2}}{\gamma^{2}}
$$

and is experimentally found to about 3 , giving a dipole moment ratio $\nu \approx 0.8 \mu$. Using the experimentally determined exciton dephasing rate in the $\left(\sigma^{+}, \sigma^{+}\right)$configuration for positive delay times of $\gamma=5 \times 10^{11} \mathrm{~s}^{-1}$, the LFE parameter corresponds to a static Lorentz shift of $\varepsilon=\hbar \widetilde{\xi}=0.07 \mathrm{meV}$.

The EID parameter is determined by the intensity ratio of the FWM traces at the $X$ transition in $(\uparrow \uparrow)$ and $(\uparrow \rightarrow)$ configuration for positive delay times. The experimentally observed intensity ratio of 40 yields $\tilde{\eta}=2.65 \times 10^{12} \mathrm{~s}^{-1}$. The calculated FWM intensities as a function of the delay-time are shown in Figs. 4(a) and 4(b) using the deduced parameters. The curve in $\left(\sigma^{+}, \sigma^{+}\right)$configuration was obtained by setting $B=0$ and 


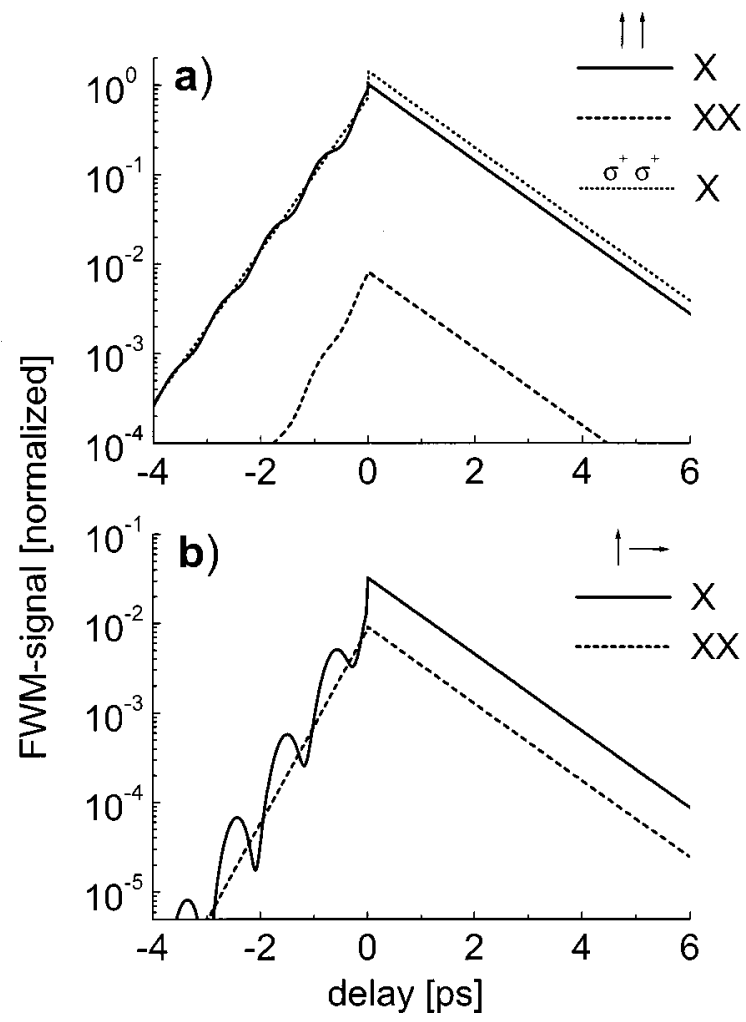

FIG. 4. (a) Calculated delay time-dependent FWM intensities at the energetic position of the $11 h$-exciton transition $(X)$ for $(\uparrow \uparrow)$ and $\left(\sigma^{+}, \sigma^{+}\right)$polarized fields and at the exciton-to-biexciton transition $(X X)$ for $(\uparrow \uparrow)$ polarized fields. (b) Calculated FWM intensities at the energetic position of the $11 \mathrm{~h}$ exciton $(X)$ and the exciton-tobiexcition transition $(X X)$ for $(\uparrow \rightarrow)$ polarized fields.

increasing the conventional FWM and the LFE terms by a factor of 2 , since the exciton-to-unbound biexciton transition is prohibited. In addition, Fig. 3(b) gives the calculated spectrally resolved FWM intensity for negative delay in $(\uparrow \uparrow)$ configuration. Except for the weak QB structures and a slightly higher decay rate in the exciton $X$ trace for negative delay and in the biexciton $X X$ trace for positive delay, the experimentally observed FWM traces are in good agreement with the calculations. The oscillations in the FWM trace for negative delay in $(\uparrow \uparrow)$ configuration can be attributed to the interference of the strong $X$ EID with the BIF contribution. Since the dephasing rate $\gamma_{b g}$ of the biexciton-to-ground-state coherence is higher than the dephasing rate $2 \gamma$ of the EID contribution the oscillations are fanning out with increasing de- lay. The oscillations vanish in the $\left(\sigma^{+}, \sigma^{+}\right)$configuration since the BIF is suppressed. In $(\uparrow \rightarrow)$ configuration the oscillations are due to the interference of the $X$ LFE with the BIF being the dominant FWM contribution in this case. The observed increase of the modulation depth with increasing negative delay is explained by the growing importance of the LFE having a dephasing rate of $2 \gamma$, which is lower than $\gamma_{b g}$. The observed slightly higher dephasing rate at the $X$ position for negative delay and at the $X X$ position for positive delay compared to the calculations is related to small inhomogeneous broadening effects which are not included in the theoretical model.

In conclusion, we have isolated the contributions of the various interaction effects in the FWM response of a nearly homogeneously broadened, quasi-two-dimensional excitonic resonance. A five-level model for the FWM response including the EID, LFE, and BIF processes has been presented to quantify the influence of the individual mechanisms. For the investigated ZnSe SQW, the EID, e.g., a spatial modulation of the dephasing by the exciton density, dominates the FWM signal in $(\uparrow \uparrow)$ configuration. For positive delay the relative FWM amplitude ratios of the EID: conventional FWM:LFE effects at the $X$ position is found to be $\tilde{\eta} / \gamma: 1: \tilde{\xi} / \gamma$ $=5.3: 1: 0.2$ using the assumption of equal dipole matrix elements for the unbound biexciton-to-exciton and for the exciton-to-ground-state transitions. For negative delay the relative FWM amplitude ratios of the EID:BIF:LFE effects is determined to $\tilde{\eta} / \gamma: B^{2}: \tilde{\xi} / \gamma=5.3: 0.64: 0.2$. In the $(\uparrow \rightarrow)$ configuration, the EID vanishes and the conventional FWM, $\mathrm{BIF}$, and the weaker LFE remain. The evaluated amplitude ratio is modified if different dipole matrix elements are considered whereas the process hierarchy is not changed. Within certain restrictions, the deduced importance of the different FWM effects can be transferred to III-V MQW structures. The reduction of LFE due to the smaller dipole moments and the lower EID parameter ${ }^{5}$ lead to a reduction of both effects in the total FWM-signal. As noted in Ref. 5, this could emphasize the importance of the biexciton-induced contributions.

We thank H. Preis and W. Gebhardt for providing the $\mathrm{Zn}_{0.9} \mathrm{Mg}_{0.1} \mathrm{Se} / \mathrm{ZnSe} \mathrm{SQW}$ structure. This work was supported by the Deutsche Forschungsgemeinschaft and by the Danish Ministries of Research and Education in the framework of CNAST. The work at the University of Iowa was supported by the U.S. Army Research Office and the National Science Foundation.

\footnotetext{
${ }^{1}$ M. Wegener et al., Phys. Rev. A 42, 5675 (1990).

${ }^{2}$ H. Wang et al., Phys. Rev. Lett. 71, 1261 (1993).

${ }^{3}$ H. Wang et al., Phys. Rev. A 49, R1551 (1994).

${ }^{4}$ Y. Z. Hu et al., Phys. Rev. B 49, 14382 (1994).

${ }^{5}$ H. Wang et al., Solid State Commun. 91, 869 (1994).

${ }^{6}$ E. J. Mayer et al., Phys. Rev. B 50, 14730 (1994).

${ }^{7}$ H. P. Wagner et al., Phys. Rev. B 59, 4584 (1999).

${ }^{8}$ T. Häupl et al., Phys. Status Solidi B 194, 219 (1996).

${ }^{9}$ T. Kuroda et al., J. Lumin. 66\&67, 429 (1996).
}

\footnotetext{
${ }^{10}$ V. Kozlov et al., Phys. Rev. B 53, 10837 (1996).

${ }^{11}$ M. Hübner et al., Phys. Rev. Lett. 76, 4199 (1996).

${ }^{12}$ A. Knorr et al., Phys. Status Solidi B 188, 473 (1995).

${ }^{13}$ M. Wörz et al., Phys. Status Solidi B 202, 805 (1997).

${ }^{14}$ H. P. Wagner et al., Phys. Rev. B 57, 1791 (1998).

${ }^{15}$ H. P. Wagner et al., Phys. Rev. B 56, 12581 (1997).

${ }^{16}$ E. J. Mayer et al., Phys. Rev. B 51, 10909 (1995).

${ }^{17}$ A. E. Paul et al., J. Opt. Soc. Am. B 13, 1016 (1996).
} 